\title{
Analyse spatio-temporelle des précipitations horaires mesurées dans le Sud-Est de la France lors de la campagne Alpex
}

\author{
Space time analysis of hourly precipitation \\ measured in the South East of France \\ during the Alpex campaign
}

\author{
J.P. Navarre et E. Nicolet
}

Centre d'Etudes de la Neige,

Domaine Universitaire, Saint-Martin-d'Hères

Dans le cadre du programme Alpex, le Centre d'études de la neige (Grenoble) a été amené à constituer un fichier de mesures de précipitations horaires pour les mois de mars et avril 1982.

Des Alpes françaises à la bordure Sud-Est du Massif Central, et de la Provence au Sud du Jura, 124 pluviographes ont été retenus à partir des réseaux de l'EDF (Grenoble), des SRAE (Aix en Provence, Lyon-Valence), de la DDE (Nimes) et de la Météorologie.

Partant de ce fichier, on a cherché à effectuer une analyse, à échelle fine, d'épisodes pluvieux : c'est-à-dire à étudier les répartitions de précipitations à l'échelle horaire et leurs évolutions au cours du temps en fonction des conditions orographiques et météorologiques.

Dans ce cadre on s'est d'abord intéressé à la variabilité spatiale des précipitations, c'est-à-dire à quantifier des structures organisées de répartition et à localiser les zones de fortes intensités en fonction du réseau analysé. Pour l'approche sur l'évolution des précipitations il s'est avéré nécessaire de mettre en œuvre des programmes de cartographie automatique. Bien que travaillant sur une courte période cette étude nous a d'abord permis de mettre au point différentes méthodologies et programmes de traitement. Dans cette communication on présentera des résultats ainsi obtenus pour deux régions très différentes: la bordure Sud-Est du Massif Central et les Alpes du Nord.

\section{Présentation du réseau}

Le domaine s'étend sur 18 départements et concerne géographiquement 3 massifs. Le fichier initial portait sur plus de 200 stations.

Certaines données étant sur support traitable directement (EDF) d'autres sous forme de données déjà dépouillées (DDE) mais bon nombre de ces mesures horaires ne se présentaient que sous forme de diagrammes. Compte tenu de divers contrôles nous n'avons gardé que 124 postes parmi lesquels figurent 2 postes du laboratoire de biologie végétale de Grenoble. La répartition géographique ( $f$ gure 1) fait ressortir l'insuffisance de points de mesures à l'intérieur des massifs alpins, par contre la bordure Sud-Est du Massif Central présente une répartition plus homogène.

\section{Principe de l'étude - Fonctions de structure}

Les études sur les distributions de précipitations à des échelles inférieures (méso ou micro échelle) ont beaucoup progressées avec l'utilisation du radar.

D'après divers travaux (Austin, House,...) on peut retenir le principe d'une organisation de moyenne échelle assez cohérente :

- des structures importantes (organisées en bandes) dont la durée de vie atteint plusieurs heures et s'étendant sur un domaine vaste (centaine de $\mathrm{kms}$ ),

- des cellules convectives de durée très courte, quelques 


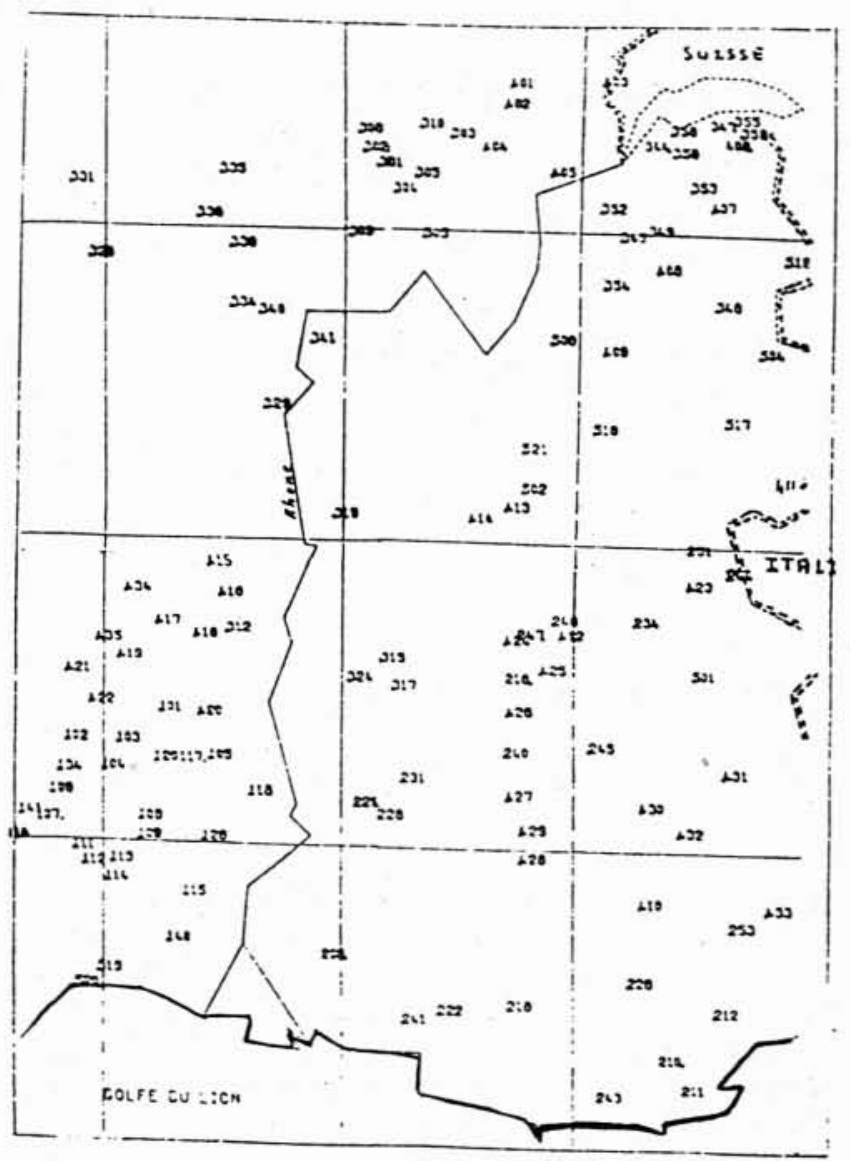

Figure 1 - Répartition géographique des stations

Le premier chiffre du code indique la provenance : 1: DDE de

Nimes; 2 : SRAE d'Aix; 3 . SRAE de Valence - Lyon; 4 : EDF; 5 : Météorologie et divers.

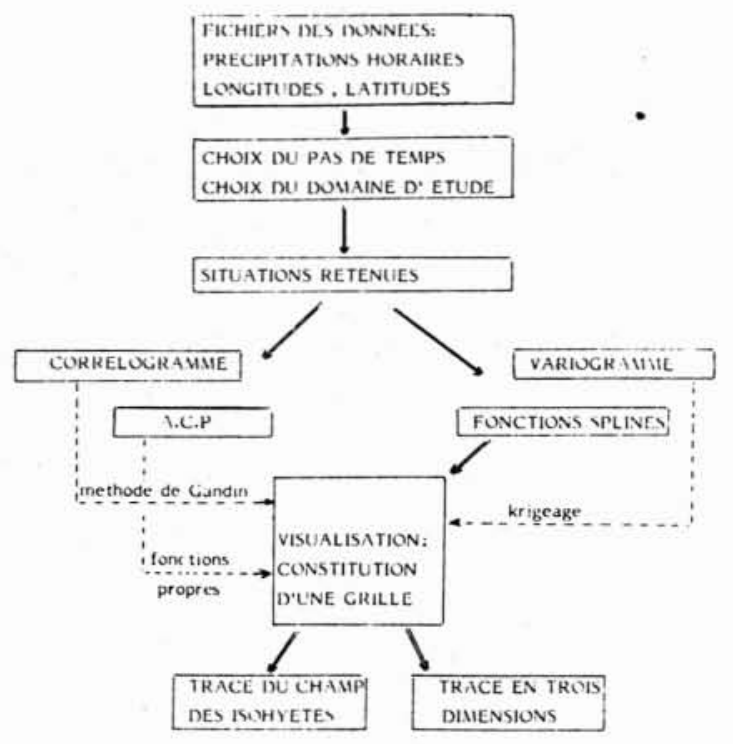

Figure 2 - Méthodologie mise en œuvre.

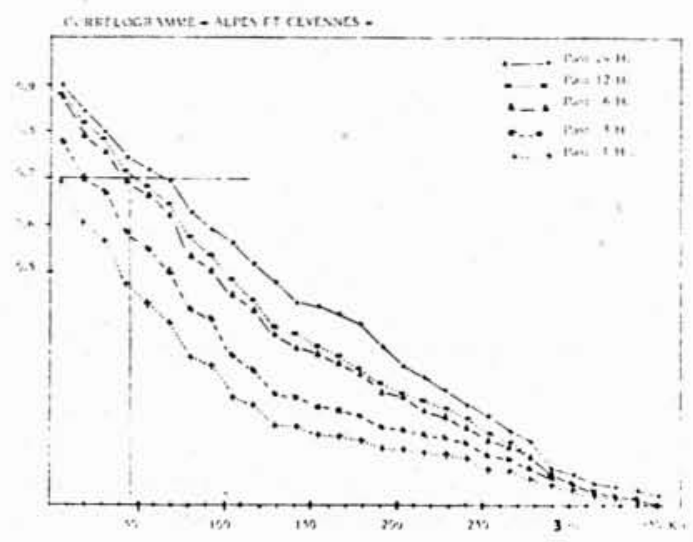

Figure 3 - Corrélogrammes.
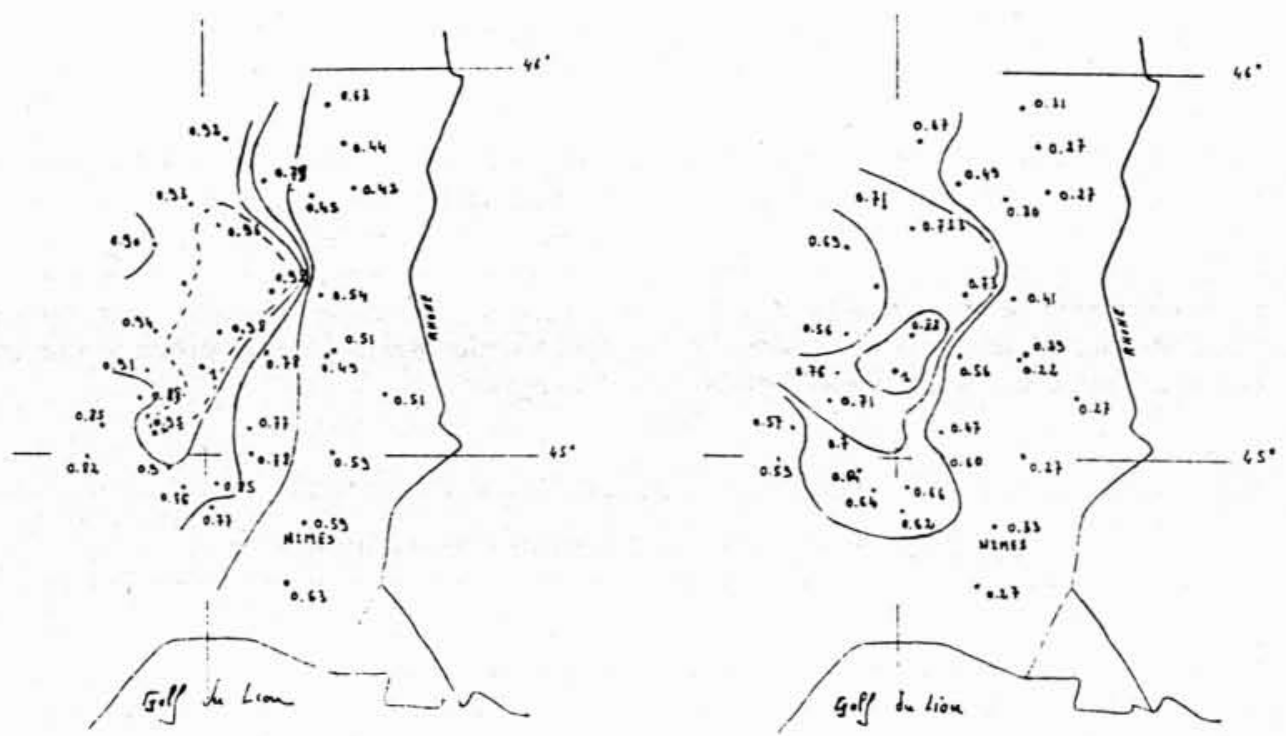

Figure 4 - Massif Central, Bordure Sud-Est. Isocorrélations. 
minutes à une heure et intéressant un domaine réduit (quelques kilomètres), observées surtout dans les trains.

Lorsqu'on veut étudier l'évolution spatio-temporelle d'un phénomène pluvieux deux approches sont possibles :

- soit on considère en différents points, l'évolution temporelle, et on aborde la structure de "l'averse ";

- soit on considère chaque image sur l'ensemble du domaine comme un signal qui se déforme au fil du temps.

Cette déformation peut présenter une certaine inertie moyenne et correspondre à des modifications homothétiques. La notion de corrélation semble alors un outil intéressant et permet alors d'apprécier l'extension et les directions principales : deux stations seront bien corrélées pour un pas de temps donné si elles restent arrosées dans un rapport constant (faiblement ou fortement simultanément). C'est le cas à l'échelle d'un épisode ou par certains types de régime; on retrouve alors une composante reproductible dans le temps.

Mais cette déformation peut apparaitre rapide ou comme un mouvement d'ensemble (essentiellement pour de faibles pas de temps) et la notion de corrélation n'est plus utilisable d'une façon brute excepté sur un domaine très limité (quelques dizaines de kilomètres).

Dans le cadre de l'étude de la variabilité spatiale on peut alors :

- soit considérer l'ensemble des observations (ou des sous ensembles) et on a une approche climatologique globale du phénomène spatial (sous réserve des conditions précédentes). On parlera de corrélogramme pour définir une fonction de structure;

- soit rechercher l'organisation à partir d'un seul champ ou pour chacun des champs : on parlera alors de variogramme pour la fonction de structure.

Ces fonctions de structure serviront alors pour une approche de la régularité spatiale de l'organisation des cellules, ce qui correspond plus à une perspective climatologique, et dans des programmes d'interpolation de champ. Les différentes étapes de l'étude et méthodes employées sont résumées sur la figure 2 .

\section{Structure spatiale des précipitations à partir des corrélogrammes}

\section{Sur l'ensemble du domaine}

Les corrélogrammes ont été tracés pour différents pas de temps de mesures: $1 \mathrm{~h}, 3 \mathrm{~h}, 8 \mathrm{~h}, 12 \mathrm{~h}, 24 \mathrm{~h}$ (figure 3 ).

Le tracé de ces divers corrélogrammes montre qu'en moyenne la décroissance, quasi-linéaire, de la corrélation avec la distance pour des pas de temps de $24 \mathrm{~h}$ et $12 \mathrm{~h}$, devient exponentielle pour $8 \mathrm{~h}$, mais surtout au pas trihoraire et horaire. Pour un seuil de 0.7 en corrélation on note comme portée (distance pour laquelle la corrélation entre 2 stations est supérieure à ce seuil)

$\begin{array}{ll}\text { Pas } 12 \mathrm{~h} & \text { Portée } 45 \mathrm{kms} \\ \text { Pas } 8 \mathrm{~h} & \text { Portée } 35-40 \mathrm{kms} \\ \text { Pas } 1 \mathrm{~h} & \text { Portée } 5-10 \mathrm{kms}\end{array}$

Compte tenu des dimensions du domaine une analyse de structure conduit donc à définir des sous régimes météorologiquement homogènes.

\section{Bordure Sud-Est du Massif Central}

Ce fichier de mars-avril 1982 n’a pas été très riche en situations de composantes Sud propres à donner des précipitations significatives sur cette région.

La distribution "moyenne" des précipitations montre l'existence d'une bande NNE/SSW avec la présence de 2 régions privilégiées: une première dans le Sud des Cévennes; une seconde sur leshauts bassins de la Cèze et de Chassesac (barrière Cévénole).

L'examen de la distribution géographique du nombre d'heures de pluie semble montrer qu'il pleut plus longtemps sur le relief qu'à l'avant, que le noyau de fortes pluies au Nord semble d'avantage le fait de précipitations persistantes et que par contre le Sud enregistre les plus fortes intensités.

On peut alors parler d'une désorganisation de la composante orographique "moyenne" des pluies sur les Cévennes. Le tracé des isocorrélations pour des cumuls de $24,8,3,1 \mathrm{~h}$ illustre ce phénomène : entre $8 \mathrm{~h}$ et $3 \mathrm{~h}$ on semble passer d'une structure de grande échelle organisée sur le relief à une organisation de moyenne échelle ( $f$ igure 4).

La représentation des corrélogrammes sur l'ensemble des 45 jours de pluie pour des cumuls de 3 et $1 \mathrm{~h}$ montre peu de variation (présence de structures persistantes de moyenne échelle) et une image qui se développe selon deux axes matérialisant la forte anisotropie des cellules de précipitations à faible pas de temps.

$\mathrm{Si}$ on trace le corrélogramme qu’à partir des seules épisodes ayant donné des précipitations dans cette région on observe alors naturellement une décroissance plus rapide avec une corrélation de 0.6 pour $4,7 \mathrm{kms}$. Si on applique au 68 champs horaires un test de signification d'un coefficient de corrélation on trouve une distance de $40 \mathrm{kms}$.

\section{Variogrammes - Bordure Sud-Est du Massif Central}

Les variogrammes définis pour chaque réalisation quantifient le degré d'amortissement des cellules de précipitations en calculant l'écart quadratique moyen entre les mesures des stations du réseau. Les tracés obtenus sur le Sud du Massif Central font apparaître un palier qui sépare une courbe quasi linéaire d'une zone fluctuante, généralement situé vers les $40 \mathrm{kms}$ pour des données quotidiennes ( $25 \mathrm{kms}-30 \mathrm{kms}$ pour des données horaires). Mais la distance n'explique que $32 \%$ de la variance spatiale; à cela deux explications : une dérive dans une direction (anisotrope) et l'effet de taille conduisant à des variations synchrones. 
Études d'épisodes affectant les Alpes du Nord

Voulant nous intéresser en premier lieu à la reconnaissance de structures "déterministes" à l'échelle d'un épisode, toutes les situations ne pouvaient être retenues et nous avons dû faire un choix; les critères de sélection retenus sont d'une part des précipitations importantes et d'autre part la présence d'un phénomène d'entrainement.

\section{Présentation des épisodes pluvieux - classification sommaire}

Travaillant sur 2 mois de mesures nous nous sommes attaché uniquement aux types de circulation générale et aux positions des centres actifs; les régimes étant ceux de l'activité pluvieuse la plus intense.

\section{Flux d'Ouest :}

4 mars 1982 : courant d'Ouest perturbé entre le $40^{\circ}$ et le $50^{\mathrm{C}}$ avec hautes pressions sur le proche Portugal.

8 avril 1982: courant d'Ouest perturbé au Nord du $45^{\circ}$ parallèle; hautes pression sur l'Espagne et I'Islande.

Flux de Nord-Ouest : (haute pression sur le Portugal)

2 mars 1982 : flux très atténué sur le Sud

20 mars 1982 : flux de Nord Ouest plus dépressionnaire.

10-11 mars 1982: avec dépression sur l'Atlantique

12-13 mars : comme précédemment mais avec un système dépressionnaire plus accentué sur la France.

\section{Flux de Sud-Ouest :}

17-18 mars 1982 : avec talweg important peu mobile sur la France.

Flux de Nord à Nord-Ouest associées à des hautes pression à proximité des îles Britanniques :

29-30-31 mars: courant dépressionnaire de composante Nord sur la France

29-30 avril : circulation de Nord - Nord-ouest

Les travaux entrepris dans cette région souligne la grande hétérogénéité dans la répartition des pluies; deux facteurs semblent prépondérant: le type de régime et l'orographie. Les pluies importantes à cette période de l'année sont toujours fortement corellées avec les advections d'air froid ou maritime.

\section{Structure spatiale des pluies enregistrées lors des divers épisodes}

\section{Régime de Nord-Ouest: étude de l'advection d'air froid}

1. Pour la situation du 2 mars, l'analyse des isohyètes au pas trihoraires montre que l'arrivée du front froid sur les Alpes correspond à une structure centrée sur les bornes et les Bauges (assez analogues à celle des précipitations de l'épisode) mais atténué sur la Chartreuse et le Vercors au premier pas.
Aux divers pas suivants, on note successivement :

- traine peu active avec noyau sur les Bauges et la Chartreuse (6-9 h)

- atténuation en Chartreuse et averses sur Vercors et Dévoluy (12-15 h)

- averses sur Dévoluy et Vercors (15-18 h

en définitif la carte quotidienne donne une bonne image de la pluviométrie associée au front froid entre 3 et $9 \mathrm{~h}$ sur les Alpes (surtout en Nord).

2. Le 20 mars, en précipitation quotidienne on retrouve une structure qui épouse l'arc Alpin de l'Est du Chablais au Nord Vercors mais l'extension sur le Sud des préalpes est moins significative.

Pour cet épisode on note au pas trihoraire l'arrivée du front chaud au Nord du domaine $(0-3 \mathrm{~h})$, puis extension aux Bornes et aux Bauches (3-6h). Entre $6 \mathrm{~h}$ et $9 \mathrm{~h}$ le front froid atteint le Lyonnais puis passe sur les Préalpes, Chartreuse et Bauges surtout et Dévoluy ( 9 h-12 h). De 12 à $15 \mathrm{~h}$ on retrouve une traine active.

Au fil des heures on retrouve des déformations ayant un axe sensiblement constant et correspondant à l'orientation de la surface frontale (et selon un entraînement à tendance Méridienne dans ce cas).

Le 12 mars, le passage du front froid induit une structure déjà rencontrée le 2 mars au moment du passage du front froid : le noyau correspond à l'arc préalpin du Sud des Bornes à la Chartreuse; la quasi-totalité des pluies tombent entre 21 et 24 heures. Mais le 13 la traine active s'étend des Bauges à l'Ouest Dévoluy une structure proche de celles du 2 mars (entre 9 et 18 h).

\section{Régime d'Ouest (peu actif) :}

Les 2 situations sont certainement non représentatives et se caractérisent par une activité faible : le noyau de précipitations n'est significatif que sur le Jura, les Bornes et le Chablais. Configuration qui se retrouve le 4 mars et le 8 avril. L'analyse de l'épisode du' 8 avril au pas trihoraire montre que le passage du front froid sur le Nord du réseau donne des précipitations significatives entre 15-18 h sur le Chablais et le Sud-Jura. Les cellules matérialisent l'orientation de la surface frontale. La zone de pluie intense progressant avec la surface frontale. Ultérieurement entre 18-21 h on retrouve une distribution frontalisée sur les Bauges avec pour les 2 pas horaires des déformations d'ensemble selon l'axe d'entraînement général qui subsiste jusqu'en fin de journée dans le Nord avec une cellule de traîne vers le Dévoluy (21-24 h).

\section{Régime de composante Nord}

Les 2 situations sont des descentes d'air froid canalisées par l'anticyclone à proximité des îles Britanniques. Les vitesses frontales sont faibles au sol et les précipitations supérieures à $10 \mathrm{~mm} / \mathrm{j}$ sont localisées sur les Dombes au Jura et au Chablais.

\section{Régime de Sud-Ouest}

Le 18 la circulation générale prend une composante Nord-Ouest après le passage de talweg sur les préalpes (noyau en Chartreuse et atténué en Dévoluy). 


\section{Analyse en composantes principales (figure 5)}

L'examen des cartes au pas trihoraire souligne des avances du système pluvieux très rapide qui semble presque discontinu. Si on passe au pas horaire, on perd une image spatiale réaliste, au profit certes, d'une approche plus réelle du phénomène d'entraînement visible que pendant les premières heures.

Mais la traine constituée d'averses sporadiques semble très difficile à apprécier.

$\mathrm{Si}$ on ne considère que des structures relativement figées, leurs évolutions n'apporte guère plus d'informations qu'à l'échelle de l'épisode. Compte tenu du réseau nous avons alors suivi une démarche consistant à essayer de dégager des structures types qui puissent se rattacher aux configurations réellement observées. Pour cela nous avons effectué une analyse en composante principale pour les 103 observations trihoraires effectuées en 57 points de l'Ubaye au Jura et aux Dombes. $90 \%$ de la variance est prise en compte par les 15 premiers axes factoriels (70\% avec 4 axes). La représentation des champs des isocosinus directeurs pour les 3 premiers composantes nous donne : pour la première composante $(\mathbf{4 0} \%)$ une surface ellipsoïde orientée NE-SW avec 3 noyaux importants : Est Chablais, Bauges-Bornes Aravis; Chartreuse Vercors.
Le second axe factoriel matérialise une structure plutôt axée W.E. avec 2 noyaux de plus fortes corrélations sur les Dombes et l'Est du Chablais avec éventuellement l'Ouest des Bornes. Le troisième axe forme une ellipsoïde NE-SW avec deux noyaux sur la partie Est des Bornes et surtout sur la partie Vercors-Chartreuse.

L'axe 4 oppose les préalpes du Sud à toutes les stations du Nord des Alpes.

\section{Conclusion}

Le fichier analysé correspond à une période beaucoup trop courte pour permettre de dégager des idées générales. Outre le développement de programmes d'analyses, il nous a cependant permis d'effectuer une étude fine de répartitions de précipitations et de mettre en valeur l'influence du pas de temps utilisés sur les résultats.

Les programmes d'interpolation par fonctions splines ont été fournis par l'Institut de Mécanique des fluides de Grenoble (section hydrologie) que nous tenons à remercier ici.
Figure 5 - Précipitations quotidiennes et trihoraires -2 mars

a - précipitations quotidiennes

b-c-d précipitations trihoraires correspondant aux périodes $0-3 \mathrm{~h} ; 3-6 \mathrm{~h}$; 6-9 h.

$\mathrm{HE}$ indique l'heure de la mesure
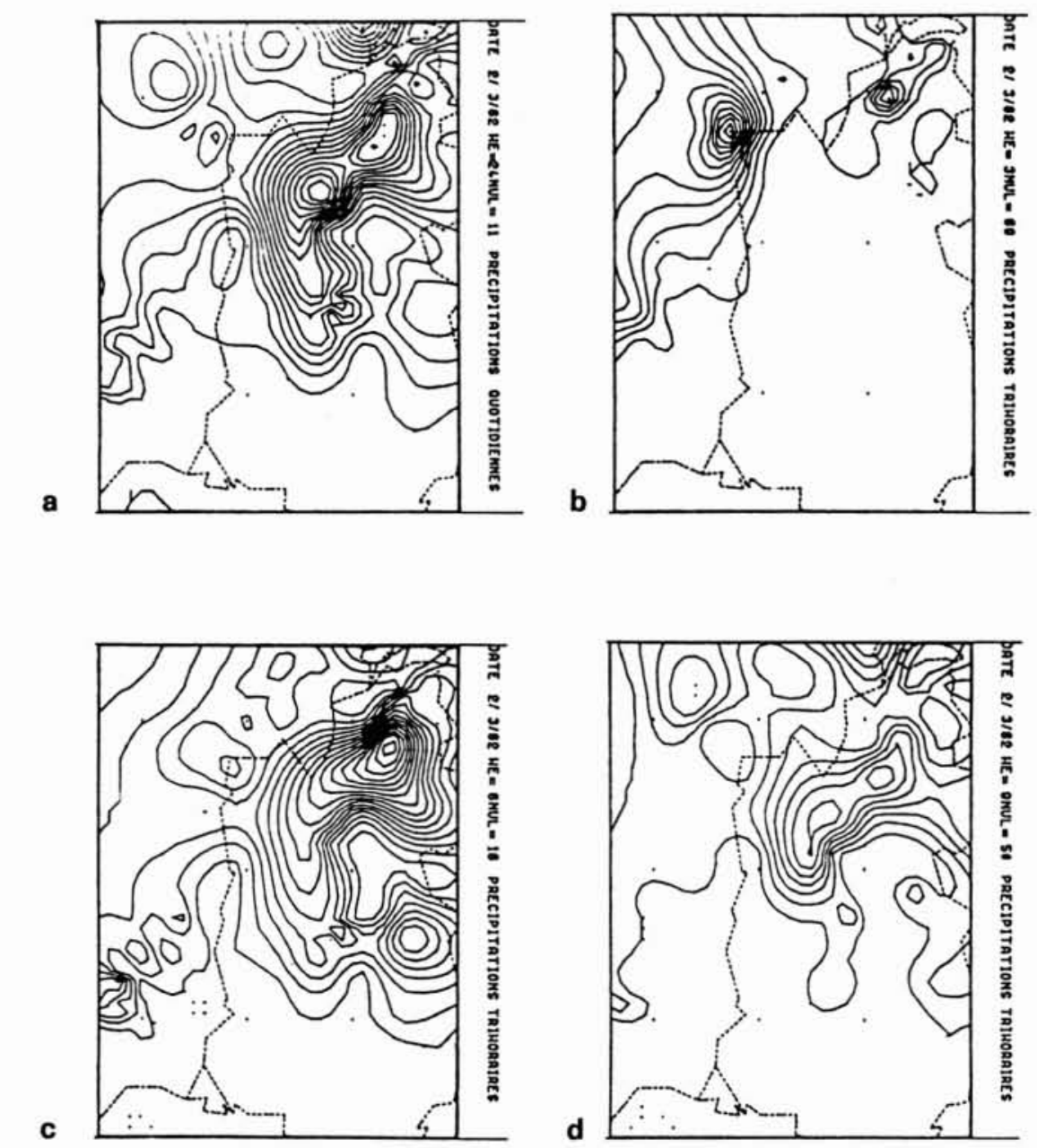
Figure 6 - Précipitations trihoraires 2 mars (suite)

a-b-c-d correspondent aux périodes 9-12h; 12-15 h; 15-18 h; 18-21 h.
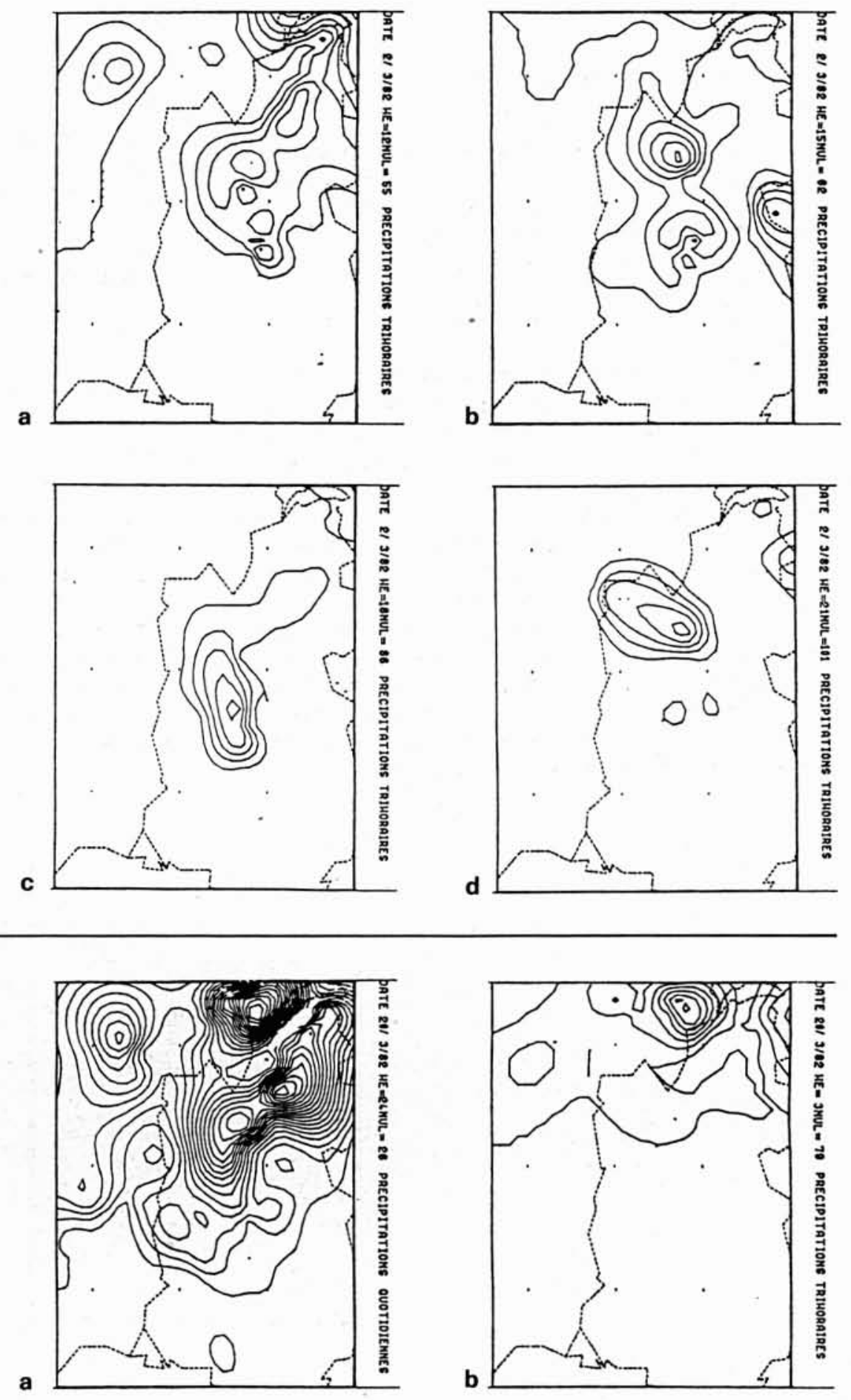

Figure 7 - Précipitations quotidiennes et trihoraires - 20 mars.
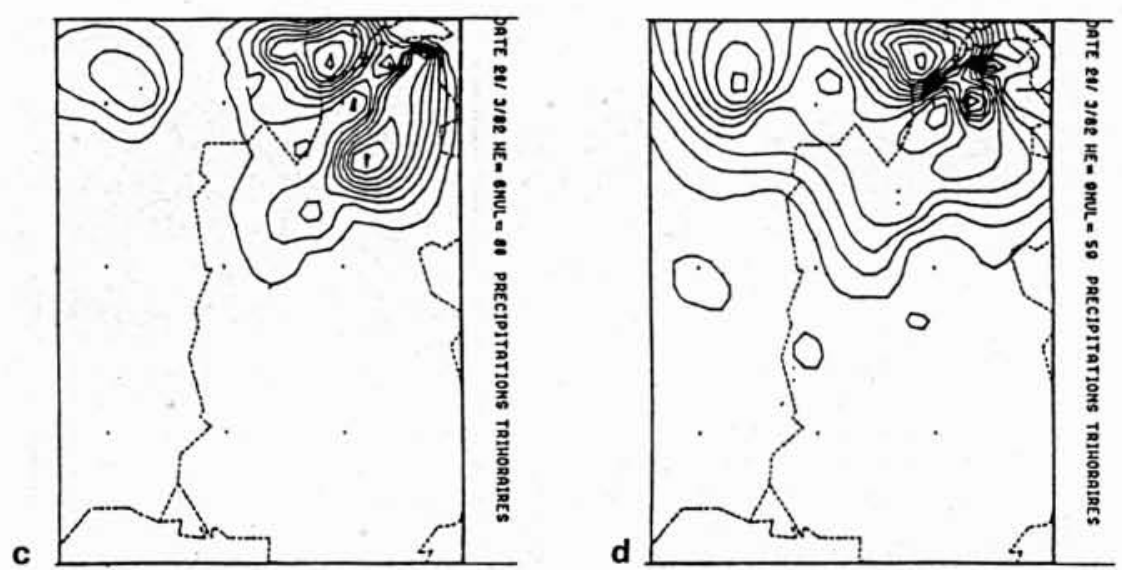

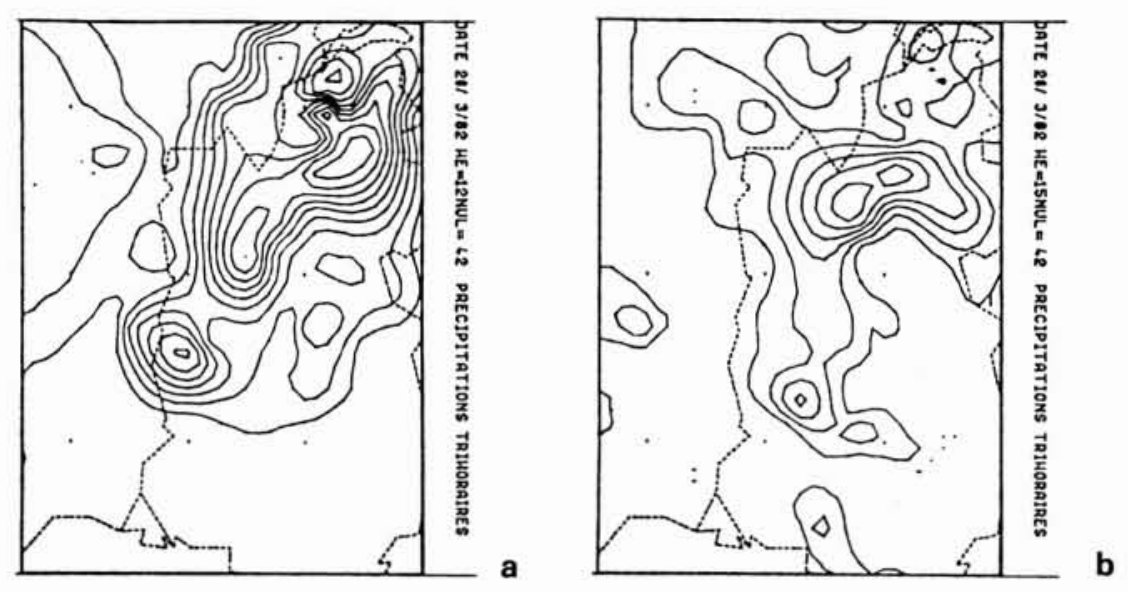

Figure 8 - Précipitations trihoraires 20 mars (suite)

a-b-c périodes $9-12 \mathrm{~h} ; 12-15 \mathrm{~h} ; 15-18 \mathrm{~h}$.
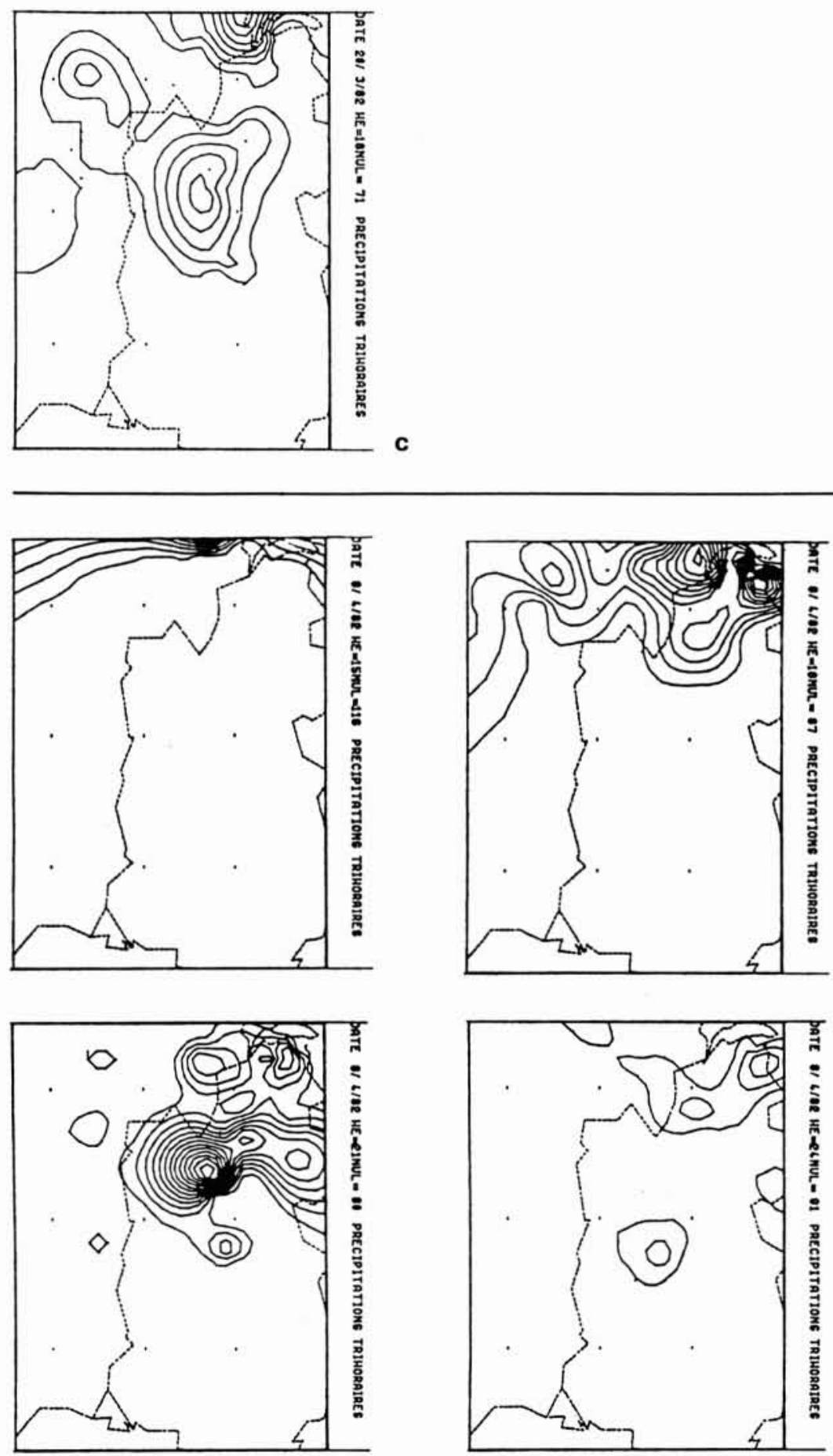

Figure 9 - Précipitations trihoraires 8 avril.

a-b-c-d périodes $12-15 \mathrm{~h}$; 15-18 h; 18-21 d $\mathrm{h} ; 21-24 \mathrm{~h}$. 


\section{Références bibliographiques}

BRAVARD A. - Contribution à la prévision des précipitations sur les Alpes du Nord à partir d'une classification des types de temps et circulation à $500 \mathrm{mb}$, Météorologie Nationale, note de I'DENM.

CREUTIN J.P. - « Méthode d'interpolation optimale de champs hydrométéorologiques. Comparaison et application à une série d'épisodes pluvieux cévénols ".

PERIO J. - La frontologie et les observations spatiales, note technique de la météorologie nationale.
TOURASSE P. - Analyses spatiales et temporelles des précipitations et utilisation opérationnelle dans un système de prévision de crues. Application aux régions cévénoles, Université scientifique et médicale de Grenoble.

Ecole National supérieure d'Hydraulique (I.N.P.G.) - Crues et précipitations intenses. Système d'annonce. Prévision. Valeurs extrêmes, Fascicule du stage de perfectionnement pour techniciens, ingénieurs et cadres.

\section{Discussion}

Président : M. L. LLIBOUTRY

M. Le Président remercie M. Navarre, en soulignant l'intérêt des tableaux des répartitions qui montrent bien ce qui se passe, et ouvre la discussion.

M. BEZINGE demande quelle est la concordance entre les données sur le terrain et les images radar. Ceci reste à faire répond M. Navarre. Mais ce développement est envisagé à l'avenir.

Pour M. ADMIRAT la ressemblance des images radar présentées avec celles recueillies en 1973 est forte : mêmes formes des isothermes nord-sud, mêmes déviations vers l'est. M. NAVARRE souligne la difficulté à comparer les répartitions quotidiennes et celles horaires.

Ceci est confirmé par $M$. BLIN qui fait état des résultats obtenus à MARIGNANE et des comparaisons avec les pluviomètres de la D.D.E.. Même à l'échelle horaire l'analyse comparative est difficile. Il faudrait pouvoir procéder à des intégrations spatiotemporelles de l'image radar digitalisée.

$M$. Le Président demande quelles seraient les recommandations pour l'emplacement des pluviographes en Maurienne ou Isère.

Effectivement, répond $M$. NAVARRE, l'lsère et la Maurienne apparaissent pauvres en pluviographes mais il $\mathbf{y}$ a aussi des problèmes de qualité d'enregistrements qui nous ont conduit à délaisser certaines mesures. Il serait certes souhaitable d'avoir une répartition plus homogène de postes de mesures, répartition qui tiendrait compte de l'orographie, tout en se méfiant des problèmes liés au vent et au chauffage des pluviographes. 